\title{
Decidability Problems for Self-induced Systems Generated by a Substitution
}

\author{
Timo Jolivet ${ }^{1}$ and Anne Siegel ${ }^{2,3(\bowtie)}$ \\ 1 Université Paul Sabatier, Institut de Mathmatique de Toulouse, Toulouse, France \\ ${ }^{2}$ CNRS, Université de Rennes 1, IRISA-UMR 6074, Rennes, France \\ anne.siegel@irisa.fr \\ 3 Inria Rennes - Bretagne Atlantique, Team Dyliss, Rennes, France
}

\begin{abstract}
In this talk we will survey several decidability and undecidability results on topological properties of self-affine or self-similar fractal tiles. Such tiles are obtained as fixed point of set equations governed by a graph. The study of their topological properties is known to be complex in general: we will illustrate this by undecidability results on tiles generated by multitape automata. In contrast, the class of self affine tiles called Rauzy fractals is particularly interesting. Such fractals provide geometrical representations of self-induced mathematical processes. They are associated to one-dimensional combinatorial substitutions (or iterated morphisms). They are somehow ubiquitous as self-replication processes appear naturally in several fields of mathematics. We will survey the main decidable topological properties of these specific Rauzy fractals and detail how the arithmetic properties of the substitution underlying the fractal construction make these properties decidable. We will end up this talk by discussing new questions arising in relation with continued fraction algorithm and fractal tiles generated by S-adic expansion systems.
\end{abstract}

The following survey is mainly inspired by three papers from the authors and their collaborators $[32,60,84]$.

\section{Substitutions Among Mathematics and Computer Science}

A substitution (sometimes also called iterated morphism) is a combinatorial object which produces sequences by iteration. It is given by a replacement rule of the letters of a finite alphabet by nonempty, finite words over the same alphabet. Thus substitutions define an iteration process on a finite set in a natural way: as we shall detail it below, they appear in many fields of mathematics, theoretical physics and computer science whenever repetitive processes or replacement rules occur.

In combinatorics on words, substitutions have been used in order to exhibit examples of finite words or infinite sequences with very specific or unusual combinatorial properties. The most famous example is the Thue-Morse sequence, 
that is, the infinite fixed-point of the substitution $\sigma(1)=12, \sigma(2)=21$, which is the first example of an overlap-free infinite sequence, meaning that it contains no subword of the shape $1 u 1 u 1$, where $u \in\{1,2\}^{*}[25,49,52,71,87,88]$. The famous class of Sturmian sequences, including the famous Fibonacci substitution, is also strongly related to the composition of substitutions in relation with continued fraction expansion (see [49, Chapter 6]). Their combinatorial properties are particularly interesting, in terms of minimal complexity as well as in terms of representation of discrete lines. A sub-class of sturmian sequences with a quadratic irrational ratio are even proved to be a fixed point of a substitution [92]. More generally, the use of substitutions to describe discrete planes in $\mathbb{R}^{3}$ has proved to be very useful in discrete geometry to decide algorithmically whether a discrete patch is a part of a given discrete plane $[12,28,48]$.

Since the time when they first appeared, substitutions have also been deeply related to number theory. For instance, in the field of diophantine approximation, substitutions produce transcendental numbers which can be approximated by cubic algebraic integers only in a very bad way [81]; the description of greedy expansions of reals in noninteger base $[5,89]$ by means of substitutions also results in best approximation characterizations [53,71]. The Cobham Theorem [41] also constitutes a strong bridge between substitutions and number theory and allows one to derive deep transcendence properties: the real numbers with continued fraction expansions given by the Thue-Morse sequence, the BaumSweet sequence [22] or the Rudin-Shapiro sequence [82] are all transcendental, the proof being based on the "substitutive" structure of these sequences [1].

Substitutions also appear in theoretical physics in connection with quasicrystals, a class of crystals with forbidden symmetry [34,72,83]. Roughly, a solid is usually considered as a quasicrystal when it has an essentially discrete diffraction diagram. From a more combinatorial point of view, a quasicrystal is given by an aperiodic but repetitive structure that plays the role of the lattice in the theory of crystalline structures. Mathematically, we then speak of Meyer sets which are obtained by employing the cut-and-project scheme [76]. In the one-dimensional case, a well-studied family of Meyer sets is given by analogs of the integers in radix representations with respect to a non-integral base, in relation with sturmian sequences [51]. In higher-dimensional cases, however, the situation becomes much more difficult. The well-known Penrose tiling is a quasicrystal since it has essentially discrete diffraction diagram, but defining a wide class of examples of quasicrystals is an open question $[10,62,63,80]$. Good candidates for cut-and-project schemes (hence, quasicrystals) are given by discrete approximations of planes that are orthogonal to Pisot directions $[11,23]$ and can be generated from one-dimensional substitutions with continued fraction algorithms $[11,56]$. Following this construction process, focusing on the periodic cases - an analog to the quadratic case within the sturmian family - it has been proved that substitutions provide relevant classes of examples for such Meyer sets in the multi-dimensional case, resulting in explicit examples of atomic structures (or point sets) with a relevant discrete diffraction diagram [30]. 
Another independent reason for the introduction of substitutions is related to dynamical systems, and more precisely to the field of symbolic dynamics, that is, the study of dynamical systems by coding their orbits as infinite sequences; to this matter, a complicated dynamics over a quite simple space is replaced by a simple dynamics (the shift mapping) over an intricate but combinatorial space made of infinite sequences. For the complete class of dynamical systems for which past and future are disjoint, the symbolic dynamical systems are particularly simple: they are described by a finite number of forbidden words, and they are called shifts of finite type [68]. A partition that induces a coding from a dynamical system onto such a shift of finite type is called a Markov partition [3]. The existence of Markov partitions is extremely useful in studying many dynamical properties (especially statistical ones); they are used for instance in analytic number theory of in dynamical systems $[4,36]$. Explicit Markov partitions, however, are generally known only for hyperbolic automorphisms of the two-dimensional torus [3], and they have rectangular shapes. In higher dimensions, a slightly different behavior appears since several results attest that the contracting boundary of a member of a Markov partition cannot be smooth $[37,40]$. In this setting, substitutions have been proved to be useful to construct explicit Markov partitions, based on generalized radix representations with a matrix as base (derived from the substitutions) [63, 65-67,77] or referring to two-dimensional iteration processes (discrete planes construction processes discussed above) $[14,55]$.

In contrast to shifts of finite type we mention highly ordered self-similar systems with zero entropy, which can be defined as systems where the large-scale recurrence structure is similar to the small-scale recurrence structure, or more precisely, as systems which are topologically conjugate to their first return mapping on a particular subset. Importantly, such dynamical systems connected to all fields described above: they appear as a return mapping of the expanding flow onto the contracting manifold for hyperbolic toral automorphisms with a unique expanding direction [20] (in relation with dynamical systems and Markov partitions); they allow one to generate infinite sequences with interesting combinatorial features (in relation with combinatorics of words); they provide a construction of points sets for quasicristals (in relation with theoretical physics); they also allow one to describe non unique greedy expansions of reals in non integer basis. From their self-similar structure, their symbolic dynamical systems are naturally generated by substitutions $[49,78]$. Therefore, substitutions appear to be ubiquitous to describe self-repetitive mathematical processes.

Paper Organization. In Sect. 2 we define Rauzy fractals and explain how they are used in the study of one-dimensional substitution dynamics. We highlight how the topological properties of these sets are used to study substitutions under several different viewpoints. In Sect. 3 we describe a general framework to study fractal objects, namely graph-directed iterated function systems (GIFS). This class of objects includes Rauzy fractals. We see that many natural topological properties are undecidable in this framework. In Sect. 4 we give several examples of properties which are undecidable in the GIFS framework, but which are in 
decidable for the particular family of Rauzy fractals, and we briefly describe the main ideas behind some of the algorithms. In Sect. 5 we consider the more general problem of understanding the infinite family of systems generated by all the products of substitutions from a given finite set, and how we can extend the existing tools to study study such families.

\section{The Geometry of One-Dimensional Substitutions}

In the world of substitutions, geometrical objects appeared in 1982 in the work of Rauzy [79], to build a domain exchange in $\mathbb{R}^{2}$ that generalizes the theory of interval exchange transformations [61,91].

To each substitution one can associate an incidence matrix $\mathbf{M}=\left(m_{i j}\right)$ in a natural way. Indeed, $m_{i j}$ counts the occurrences of the letter $i$ in $\sigma(j)$. To build a Rauzy fractal, we restrict to the case of unit Pisot substitutions, i.e., substitutions whose incidence matrix is primitive and has a Pisot unit as a dominant eigenvalue.

An approach to build a Rauzy fractal is based on formal power series and projections of broken lines to hyperplanes and is inspired by Rauzy's seminal paper [79]. The principle is to consider a periodic point for the substitution, then to represent this sequence as a stair (also called "broken line") in $\mathbb{R}^{n}$, where $n$ denotes the size of the alphabet on which the substitution acts. The next step is to project the vertices of the stair onto the contracting subspace of the incidence matrix. Since the projection is performed on a contracting stable space of the matrix, and the object that was projected is a periodic point of the substitution (and, hence, "contracted" by the incidence matrix) the closure of the projection is a compact set. A final step consists in drawing several colors with respect to the direction used in the stair to arrive on each vertex before the projection, and we get the Rauzy fractal.

The standard example is given by the so-called Tribonacci substitution defined as $\sigma(1)=12, \sigma(2)=13, \sigma(3)=1$ which was first studied by Rauzy [79]. Projecting the "broken line" related to the unique fixed point of the Tribonacci substitution to the two-dimensional contracting plane yields a nice fractal picture, the so-called classical Rauzy fractal $\mathcal{T}$ which is depicted in Fig. 1 with its subtiles $\mathcal{T}(1)$ (largest subtile), $\mathcal{T}(2)$ (middle size subtile), $\mathcal{T}(3)$ (smallest subtile).

Since this compact set is obtained from the fixed point of the substitution, the self-replication properties of the fixed point have geometrical consequences: we represent the contracting space as the complex plane $\mathbb{C}$. Denote by $\alpha$ one of the two complex conjugate roots of the characteristic polynomial of the substitution matrix $X^{3}-X^{2}-X-1$; one has $|\alpha|<1$. With help of $\alpha$, the Rauzy fractal can be written as graph directed iterated function system in the sense of [73] as

$$
\left\{\begin{array}{l}
\mathcal{T}(1)=\alpha(\mathcal{T}(1) \cup \mathcal{T}(2) \cup \mathcal{T}(3)), \\
\mathcal{T}(2)=\alpha \mathcal{T}(1)+1 \\
\mathcal{T}(3)=\alpha \mathcal{T}(2)+1
\end{array}\right.
$$



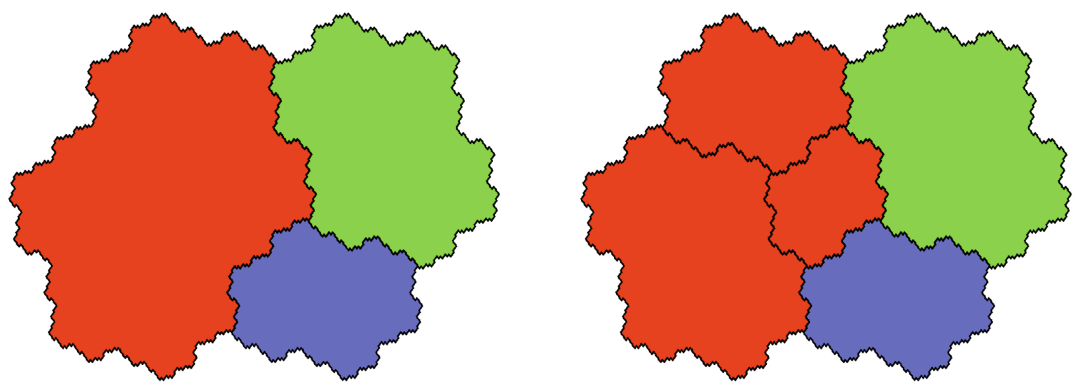

Fig. 1. The classical Rauzy fractal with its subtiles (left), and their self-affine decompositions (left).

Hence, each subtile is a finite union of translated contracted copies of subtiles. The contraction is given by the Galois conjugate $\alpha$ (of modulus $<1$ ), while the translations depend on the structure of the substitution.

The main reason for the frequent use of Rauzy fractals in the literature is that the iterative procedure to generate infinite words with the help of a substitution is often shifted to a geometric framework and reflects in self-similarity properties that can be studied. Then, the main questions to be investigated in each domain can be interpreted as questions related to the topology of the Rauzy fractal and its tiling properties.

In number theory, diophantine properties are induced by properties of a distance function to a specific broken line [53] related to the Rauzy fractal and the size of the largest ball contained in it. Finiteness properties of digit representations in numeration systems with non-integer base are related to the fact that $\mathbf{0}$ is an inner point of the Rauzy fractal [9]. More generally, the identification of those real numbers who has a periodic expansion in non-integer basis is strongly related to the study of the intersection of the fractal boundary with appropriate lines $[2,6]$. Rauzy fractals also allow one to characterize purely periodic orbits of representations in numeration systems w.r.t. non-integer base, and yield certain generalizations of Galois' theorem $[27,30,57]$. In discrete geometry, there are numerous relations between generalized Rauzy fractals and discrete planes as studied for instance in [13]. The shape of pieces generating a discrete plane is tightly related to the shape of Rauzy fractals. On the dynamical system viewpoint, Rauzy fractals allow one to explicitly build the largest spectral factor induced by a substitutive dynamical system. Explicit Markov partitions for hyperbolic automorphisms of tori are constructed for instance in [55,77], actually using Rauzy fractals. Importantly, in this setting, connectivity properties of Rauzy fractals are crucial to establish generator properties of the Markov partition [3]. In tiling theory, Rauzy fractals are used to represent the tiling flow and to prove that substitutive systems are expanding foliations of the space tiling [17].

For all these reasons, a thorough study of the topological properties of Rauzy fractals have appeared to be of great importance. There have been quite many 
contributions on this field in the last decade, establishing that many topological properties are semi-decidable. In the following, we will illustrate why such decidability results are somehow quite unexpected with respect to the most general study of self-affine tiles.

\section{Undecidability of GIFS Topological Properties}

In order to investigate more formally the topological properties of Rauzy fractals, we will consider these compact sets in the larger family of graph-directed function systems (GIFS).

One of the most common ways to define fractals is to use an iterated function system (IFS), defined by a finite collection of maps $f_{1}, \ldots, f_{n}: \mathbb{R}^{d} \rightarrow \mathbb{R}^{d}$ which are all contracting: there exists $0 \leq c<1$ such that $\left\|f_{i}(x)-f_{i}(y)\right\| \leq c\|x-y\|$ for all $x, y \in \mathbb{R}^{d}$. The associated fractal, called the attractor of the IFS, is the unique nonempty compact set $R$ such that

$$
R=\bigcup_{i=1}^{n} f_{i}(R) .
$$

Such a set $R$ always exists and is unique thanks to a famous result of Hutchinson [54], based on an application of Banach fixed-point theorem; see also [46] or [21]. For example, the classical Cantor set can be defined as the unique compact set $X \subseteq \mathbb{R}$ satisfying the set equation $X=\frac{1}{3} X \cup\left(\frac{1}{3} X+\frac{2}{3}\right)$, and the Sierpiński triangle can defined as the unique compact set $X \subseteq \mathbb{R}^{2}$ satisfying $X=\frac{1}{2} X \cup\left(\frac{1}{2} X+(1 / 2,0)\right) \cup\left(\frac{1}{2} X+(0,1 / 2)\right)$.

A natural generalization of IFS can be obtained by restricting which infinite sequences of maps $\left(f_{i_{n}}\right)_{n \in \mathbb{N}}$ we are allowed to iterate. One of the simplest such restrictions is to require the set of allowed sequence $\left(i_{n}\right)_{n \in \mathbb{N}}$ to be the language of the infinite paths of a finite graph. Doing so we can give a new definition: a $d$-dimensional graph-directed iterated function system (GIFS) [73] is a directed graph in which each edge $e$ is labelled by a contracting mapping $f_{e}: \mathbb{R}^{d} \rightarrow \mathbb{R}^{d}$.

It can be shown by a fixed point argument that given a GIFS $\left(G,\left\{f_{e}\right\}_{e \in E}\right)$ there exists a unique collection of non-empty compact sets $\left\{R_{q}\right\}_{q \in \mathcal{Q}}$ such that

$$
R_{q}=\bigcup_{q \in \mathcal{Q}} \bigcup_{e \in E_{q, r}} f_{e}\left(R_{r}\right)
$$

where $\mathcal{Q}$ is the set of vertices of the directed graph defining the GIFS, and $E_{q, r}$ denote the set of edges from vertex $q$ to vertex $r$. The sets $R_{i}$ are called GIFS attractors or solutions of the GIFS. Note that the uniqueness statement does not hold for general sets, but only for non-empty compact sets [45].

In other words, the GIFS attractors are the solution of a set equation. Indeed, we are in presence of a collection of finitely many compact sets $\left\{R_{1}, \ldots, R_{q}\right\}$ such that each set $R_{i}$ can be decomposed as a union of contracted copies of itself and the other sets $R_{j}$. 
Many works are focused on the more specific family of self-affine attractors, in which the contractions $f_{i}$ must be affine (of the form $M_{i} x+v_{i}$ where $M_{i}$ is a $d \times d$ matrix and $v_{i} \in \mathbb{R}^{d}$ ), or the even more constrained family of selfsimilar attractors, in which the $f_{i}$ must be similarities (of the form $a x+v_{i}$ where $a \in[0,1]$ and $\left.v_{i} \in \mathbb{R}^{d}\right)$.

Self-affine attractors are intensively studied, and many results are known about some particular families. For example the Hausdorff dimension of BedfordMcMullen carpets admits an exact simple formula [24,74], and similar results about the fractal dimension or the Lebesgue measure of some other classes exist $[18,35,47,50,64]$. Moreover, there is an "almost sure" formula for the packing and Hausdorff dimension in the self-similar case [44].

Despite all the positive results stated above, the notorious difficulty of selfaffine sets suggests that there cannot exist any simple criteria to decide such properties in full generality. From a computer-theoretical point of view, this would correspond to undecidability results of the type: "there cannot be an algorithm that, given input an IFS specified by rational coefficients, determines if Property X holds for the IFS attractor", where "Property X" can be any IFS attractor property we are interested in. One could naturally expect a Rice-like theorem stating that every nontrivial property of GIFS attractor is undecidable, but such a statement does not hold. For example, the property of being equal to a singleton is nontrivial and decidable.

A first undecidability result has been established by Dube [42]: it is undecidable if the attractor of a rational 2-dimensional affine IFS intersects the diagonal $\{(x, x): x \in[0,1]\}$.

In [60], the undecidability of some topological properties of self-affine graphdirected iterated function systems is established. More precisely, the authors prove that deciding whether the attractor of a 2-dimensional, 3-state affine GIFS has an empty interior is undecidable. In addition, deciding whether the intersection of two GIFS attractors $R_{q} \cap R_{q^{\prime}}$ has empty interior is also undecidable.

To do so, the authors rely on the approach of Dube [42] and associate selfaffine sets with computational devices called multitape automata, which are finite automata acting on several tapes, with an independent head reading each tape. Then they relate some properties of the automaton with topological properties of its associated attractor, and they obtain the undecidability of the latter by proving the undecidability of the former.

\section{Decidability of Rauzy Fractals Properties}

Equation 2 shows that Rauzy fractals also belong to the class of GIFS. In general, a Rauzy fractal associated with a substitution $\sigma$ on the alphabet $\mathcal{A}$ satisfies the set equation:

$$
\forall i \in A, \mathcal{T}(i)=\bigcup_{\substack{j \in A, \sigma(j)=p i s}} h(\mathcal{T}(j))+\pi(p) .
$$

Where $h$ is a contraction map and $\pi$ a mapping from $\mathcal{A}^{*}$ to the Euclidean space where the fractal lives. The graph with nodes in $\mathcal{A}$ and with edges described by 
the relation $\sigma(j)=$ pis is the so-called prefix-suffix graph. It describes the way images of letters under $\sigma$ can be decomposed [38,39]. The mappings in the GIFS are contracting, thus the nonempty compact sets $\mathcal{T}(i)$ satisfying Eq. 2 (and more generally Eq. 1) are uniquely determined [73]. They always satisfy the following properties:

- The direction of the expanding eigenvector of the incidence matrix is irrational [39].

- $\mathcal{T}$ as well as $\mathcal{T}(i)$ is compact [73].

- $\mathcal{T}$ as well as $\mathcal{T}(i)$ is the closure of its interior and has a non-zero measure $[32,86]$.

- The subtiles $\mathcal{T}(i)$ induce a self-replicating multiple tiling of the contracting plane [15].

Similarly to the families studied in [44], the Hausdorff dimension of the boundary of a planar Rauzy fractal can be computed if its corresponding Pisot eigenvalue has complex conjugates, because the associated GIFS is then selfsimilar [84]. However, no formula is known if the two conjugates of the Pisot eigenvalue are real, because then their norms are not equal, so the GIFS is selfaffine but not self-similar. This is in agreement with the known difficulty of the study of self-affine sets.

On the contrary, the study of inner points and intersection between tiles is at the opposite of multitape automata: indeed, there are several algorithms to decide if the tiles of the Rauzy fractal of a unimodular Pisot substitution $\sigma$ do not overlap, that is, if they intersect on a set of Lebesgue measure zero. In the case of Rauzy fractals, this is equivalent to having intersection with empty interior. It follows that the undecidable property that is stated for 2-dimensional, 3-state affine GIFS [60] is actually decidable for the case of Rauzy fractals (see the review in [32]). This is not contradictory since the family of Rauzy fractals GIFS is disjoint from the family of the GIFS associated with multitape automata for which undecidability results are proved. Indeed, negative powers of integers cannot be the expansion factors of a Rauzy fractal GIFS, which, in opposition, is always the case for multitape automata GIFS.

More generally, it has been proved that many topological properties or Rauzy fractals are actually decidable.

- Checking whether the origin is an inner point of $\mathcal{T}$ is decidable [84].

- Checking whether the Rauzy fractal generates self-similar tiling of the plane is decidable $[7,8,17,43,58,69,70,85]$.

- The box-counting dimension of the fractal boundary of the Rauzy fractal and its subtiles is computable. In the self-similar case, this allows computing the Haussorf-dimension [84, 90].

- Checking the connectivity of $\mathcal{T}$ and $\mathcal{T}(i)$ is semi-decidable.

- Verifying that $\mathcal{T}(i)$ is homeomorphic to a closed disk is semi-decidable.

- The non-triviality of the fundamental group of $\mathcal{T}$ is also semi-decidable, as well as the property of uncountability and being not free. 
The underlying idea in all criteria is to match the structure of the graph directed iterated function system that defines the central tile with its tiling properties. All criteria make use and are expressed in terms of graphs. The graphs we are using to formulate and prove such results contain the structure of intersections of two or more tiles in the (multiple) tilings induced by the Rauzy fractal $\mathcal{T}$ and its subtiles $\mathcal{T}(i)(1 \leq i \leq n)$. If the subtiles induce a tiling, they provide a description of the boundaries of the subtiles $\mathcal{T}(i)(1 \leq i \leq n)$ and even permit to draw these boundaries in an easy way. Other graphs encode the connectivity of the Rauzy fractal, its subtiles as well as of certain pieces of their boundary.

\section{Extending the Framework of Rauzy Fractals}

As detailed below, topological properties of Rauzy fractals are now well understood and can be checked for each single Pisot unit substitution. Nonetheless, these decidability results rely on the construction of graphs which are deeply dependent on the combinatorics of the substitution and the algebraic properties of its incidence matrix. This raises a strong issue when one wishes to address general results about families of substitutions. Then, the decidability issue becomes: Is there an algorithm that, given a finite family of substitutions, determines whether Property $X$ holds for the Rauzy fractal of every finite product of substitutions in the input family of substitutions? This is for example how Arnoux-Rauzy substitutions are constructed [16]. More generally, two-dimensional continued fraction algorithms (Brun and Jacobi-Perron continued fraction algorithms) defined as piecewise fractional maps produce product families of three-letter substitutions which seems to have relevant invariant topological properties [26].

To address such decidability questions about product families of substitutions, new frameworks need to be developed. Two main trends are studied nowadays. First, relying on the mathematical study of proximality and homoclinic return points in tiling flows, Barge proved the product family of $\beta$-substitutions, Brun substitutions and Jacobi-Perron substitutions all generate aperiodic tilings, meaning that their boundary can be approximated as the Haussdorf limit of polygonal transformations [19]. Second, the construction and study of local twodimensional substitution rules to generate Rauzy fractals allow to study generic topological properties: for instance, these techniques allow one to solve the decidability problem of connectivity for Rauzy fractals associated to the product families of Arnoux-Rauzy, Brun and Jacobi-Perron substitutions (which is decidable), and simple-connectivity (which in non-decidable) [27,59]. As an application of this result, it becomes possible to elucidate which two-dimensional toral translation can be represented by a symbolic substitutive system, an extension of the representation of one-dimensional toral translation by sturmian sequences. Such a framework has also been successfully applied to a problem in discrete geometry about the critical thickness at which an arithmetic discrete plane is 2-connected [29]. 
Another raising issue is the study of fractal tiles generated by substitutionlike processes although they do not exactly fit with the framework of Rauzy fractals or GIFS. In this trend, we mention tiles generated by non unit numbers [75] or SRS-numerations systems [31], that have intricate properties, and tiles generated by infinite compositions of substitution (S-adic) framework [33]. In both cases, all remains to be done in terms of decidability problems. Indeed, in most cases, the basis algebraic properties of the underlying systems (i.e., rational independency of translation vectors in the GIFS), or the basic topological properties (i.e. the fractal has a non empty interior, 0 is a inner point) cannot be established in general. Such properties will deserve algorithmic studies and pave the way to very exciting novel issues to address.

\section{References}

1. Adamczewski, B., Bugeaud, Y., Davison, L.: Continued fractions and transcendental numbers. Ann. Inst. Fourier (Grenoble) 56(7), 2093-2113 (2006). (Numération, pavages, substitutions)

2. Adamczewski, B., Frougny, C., Siegel, A., Steiner, W.: Rational numbers with purely periodic $\beta$-expansion. Bull. Lond. Math. Soc. 42(3), 538-552 (2010)

3. Adler, R.L.: Symbolic dynamics and Markov partitions. Bull. Amer. Math. Soc. (N.S.) 35(1), 1-56 (1998)

4. Adler, R.L., Weiss, B.: Similarity of automorphisms of the torus. Memoirs of the American Mathematical Society, No. 98. American Mathematical Society, Providence, R.I (1970)

5. Akiyama, S.: Pisot numbers and greedy algorithm. In: Number theory (Eger, 1996), pp. 9-21. de Gruyter, Berlin (1998)

6. Akiyama, S., Barat, G., Berthé, V., Siegel, A.: Boundary of central tiles associated with Pisot beta-numeration and purely periodic expansions. Monatsh. Math. 155(3-4), 377-419 (2008)

7. Akiyama, S., Lee, J.-Y.: Algorithm for determining pure pointedness of self-affine tilings. Adv. Math. 226(4), 2855-2883 (2011)

8. Akiyama, S., Lee, J.-Y.: Overlap coincidence to strong coincidence in substitution tiling dynamics. Eur. J. Combin. 39, 233-243 (2014)

9. Akiyama, S., Scheicher, K.: Intersecting two-dimensional fractals with lines. Acta Sci. Math. (Szeged) 71(3-4), 555-580 (2005)

10. Anderson, J., Putnam, I.: Topological invariants for substitution tilings and their associated $C^{*}$-algebras. Ergodic Theory Dyn. Syst. 18, 509-537 (1998)

11. Arnoux, P., Berthé, V., Ei, H., Ito, S.: Tilings, quasicrystals, discrete planes, generalized substitutions, and multidimensional continued fractions. In: Discrete models: combinatorics, computation, and geometry (Paris, 2001). Discrete Math. Theor. Comput. Sci. Proc., AA, pages 059-078 (electronic). Maison Inform. Math. Discrèt. (MIMD), Paris (2001)

12. Arnoux, P., Berthé, V., Fernique, T., Jamet, D.: Functional stepped surfaces, flips, and generalized substitutions. Theor. Comput. Sci. 380(3), 251-265 (2007)

13. Arnoux, P., Berthé, V., Ito, S.: Discrete planes, $\mathbb{Z}^{2}$-actions, Jacobi-Perron algorithm and substitutions. Ann. Inst. Fourier 52(2), 305-349 (2002)

14. Arnoux, P., Furukado, M., Harriss, E., Ito, S.: Algebraic numbers, group automorphisms and substitution rules on the plane. Trans. Amer. Math. Soc. (2009, to appear) 
15. Arnoux, P., Ito, S.: Pisot substitutions and Rauzy fractals. Bull. Belg. Math. Soc. Simon Stevin 8(2), 181-207 (2001)

16. Arnoux, P., Rauzy, G.: Représentation géométrique de suites de complexité $2 n+1$. Bull. Soc. Math. Fr. 119(2), 199-215 (1991)

17. Baker, V., Barge, M., Kwapisz, J.: Geometric realization and coincidence for reducible non-unimodular Pisot tiling spaces with an application to beta-shifts. Ann. Inst. Fourier 56(7), 2213-2248 (2006)

18. Barański, K.: Hausdorff dimension of the limit sets of some planar geometric constructions. Adv. Math. 210(1), 215-245 (2007)

19. Barge, M.: Pure discrete spectrum for a class of one-dimensional substitution tiling systems (2014)

20. Barge, M., Kwapisz, J.: Geometric theory of unimodular Pisot substitutions. Amer. J. Math. 128(5), 1219-1282 (2006)

21. Barnsley, M.F.: Fractals Everywhere, 2nd edn. Academic Press Professional, Boston (1993)

22. Baum, L.E., Sweet, M.M.: Continued fractions of algebraic power series in characteristic 2. Ann. Math. (2) 103(3), 593-610 (1976)

23. Béal, M.-P., Perrin, D.: Symbolic dynamics and finite automata. In: Rozenberg, G., Salomaa, A. (eds.) Handbook of Formal Languages. Linear Modeling: Background and Application, pp. 463-506. Springer, Heidelberg (1997)

24. Bedford, T.: Crinkly curves, Markov partitions and box dimensions in self-similar sets. Ph.D. thesis, University of Warwick (1984)

25. Berstel, J., Perrin, D.: The origins of combinatorics on words. Eur. J. Comb. 28(3), 996-1022 (2007)

26. Berthé, V.: Multidimensional Euclidean algorithms, numeration and substitutions. Integers, 11B: Paper No. A2, 34 (2011)

27. Berthé, V., Bourdon, J., Jolivet, T., Siegel, A.: A combinatorial approach to products of Pisot substitutions. Ergodic Theory and Dynamical Systems (2015, to appear)

28. Berthé, V., Fernique, T.: Brun expansions of stepped surfaces. Discrete Math. 311(7), 521-543 (2011)

29. Berthé, V., Jamet, D., Jolivet, T., Provençal, X.: Critical connectedness of thin arithmetical discrete planes. In: Gonzalez-Diaz, R., Jimenez, M.-J., Medrano, B. (eds.) DGCI 2013. LNCS, vol. 7749, pp. 107-118. Springer, Heidelberg (2013)

30. Berthé, V., Siegel, A.: Tilings associated with beta-numeration and substitutions. Integers 5, 46 (2005)

31. Berthé, V., Siegel, A., Steiner, W., Surer, P., Thuswaldner, J.M.: Fractal tiles associated with shift radix systems. Adv. Math. 226(1), 139-175 (2011)

32. Berthé, V., Siegel, A., Thuswaldner, J.M.: Substitutions, Rauzy fractals, and tilings. In: Combinatorics, Automata and Number Theory. Encyclopedia of Mathematics and its Applications, vol. 135. Cambridge University Press (2010)

33. Berthé, V., Steiner, W., Thuswaldner, J.: Geometry, dynamics, and arithmetic of $S$-adic shifts. Article submitted for publication (2014)

34. Bombieri, E., Taylor, J.E.: Which distributions of matter diffract? An initial investigation. J. Phys. 47(7, Suppl. Colloq. C3), C3-19-C3-28 (1986). (International workshop on aperiodic crystals, Les Houches (1986))

35. Bondarenko, I.V., Kravchenko, R.V.: On Lebesgue measure of integral self-affine sets. Discrete Comput. Geom. 46(2), 389-393 (2011)

36. Bowen, R.: Equilibrium States and the Ergodic Theory of Anosov Diffeomorphisms, vol. 470. Springer, Heidelberg (1978) 
37. Bowen, R.: Markov partitions are not smooth. Proc. Amer. Math. Soc. 71(1), 130-132 (1978)

38. Canterini, V., Siegel, A.: Automate des préfixes-suffixes associé à une substitution primitive. J. Théor. Nombres Bordeaux 13(2), 353-369 (2001)

39. Canterini, V., Siegel, A.: Geometric representation of substitutions of Pisot type. Trans. Am. Math. Soc. 353(12), 5121-5144 (2001)

40. Cawley, E.: Smooth Markov partitions and toral automorphisms. Ergodic Theory Dyn. Syst. 11(4), 633-651 (1991)

41. Cobham, A.: Uniform tag sequences. Math. Syst. Theory 6, 164-192 (1972)

42. Dube, S.: Undecidable problems in fractal geometry. Complex Syst. 7(6), 423-444 (1993)

43. Ei, H., Ito, S., Rao, H.: Atomic surfaces, tilings and coincidences II. reducible case. Ann. Inst. Fourier 56, 2285-2313 (2006)

44. Falconer, K.: The Hausdorff dimension of self-affine fractals. Math. Proc. Camb. Philos. Soc. 103(2), 339-350 (1988)

45. Falconer, K.: Techniques in Fractal Geometry. Wiley, Chichester (1997)

46. Falconer, K.: Fractal Geometry, Mathematical Foundations and Applications, 2nd edn. Wiley, Hoboken (2003)

47. Feng, D.-J., Wang, Y.: A class of self-affine sets and self-affine measures. J. Fourier Anal. Appl. 11(1), 107-124 (2005)

48. Fernique, T.: Generation and Recognition of digital planes using multi-dimensional continued fractions. In: Coeurjolly, D., Sivignon, I., Tougne, L., Dupont, F. (eds.) DGCI 2008. LNCS, vol. 4992, pp. 33-44. Springer, Heidelberg (2008)

49. Fogg, N.P., Berthé, V., Ferenczi, S., Mauduit, C., Siegel, A. (eds.): Substitutions in Dynamics, Arithmetics and Combinatorics, vol. 1794. Springer, Heidelberg (2002)

50. Fraser, J.M.: On the packing dimension of box-like self-affine sets in the plane. Nonlinearity 25(7), 2075-2092 (2012)

51. Gazeau, J.-P., Verger-Gaugry, J.-L.: Geometric study of the beta-integers for a Perron number and mathematical quasicrystals. J. Théor. Nombres Bordeaux 16(1), 125-149 (2004)

52. Hedlund, G.A.: Remarks on the work of Axel Thue on sequences. Nordisk Mat. Tidskr. 15, 148-150 (1967)

53. Hubert, P., Messaoudi, A.: Best simultaneous Diophantine approximations of Pisot numbers and Rauzy fractals. Acta Arith. 124(1), 1-15 (2006)

54. Hutchinson, J.E.: Fractals and self-similarity. Indiana Univ. Math. J. 30(5), 713$747(1981)$

55. Ito, S., Ohtsuki, M.: Modified Jacobi-Perron algorithm and generating Markov partitions for special hyperbolic toral automorphisms. Tokyo J. Math. 16(2), 441$472(1993)$

56. Ito, S., Ohtsuki, M.: Parallelogram tilings and Jacobi-Perron algorithm. Tokyo J. Math. 17(1), 33-58 (1994)

57. Ito, S., Rao, H.: Purely periodic $\beta$-expansion with Pisot base. Proc. Am. Math. Soc. 133, 953-964 (2005)

58. Ito, S., Rao, H.: Atomic surfaces, tilings and coincidences I. Irreducible case. Isr. J. Math. 153, 129-155 (2006)

59. Jolivet, T., Kari, J.: Consistency of multidimensional combinatorial substitutions. In: Hirsch, E.A., Karhumäki, J., Lepistö, A., Prilutskii, M. (eds.) CSR 2012. LNCS, vol. 7353, pp. 205-216. Springer, Heidelberg (2012)

60. Jolivet, T., Kari, J.: Undecidable properties of self-affine sets and multi-tape automata. In: Csuhaj-Varjú, E., Dietzfelbinger, M., Ésik, Z. (eds.) MFCS 2014, Part I. LNCS, vol. 8634, pp. 352-364. Springer, Heidelberg (2014) 
61. Keane, M.: Interval exchange transformations. Math. Z. 141, 25-31 (1975)

62. Kellendonk, J., Putnam, I.: Tilings, $C^{*}$-algebras, and $K$-theory. In: Baake, M., Moody, R.V. (eds.) Directions in Mathematical Quasicrystals. AMS CRM Monogr. Ser., vol. 13, pp. 177-206, Providence, RI (2000)

63. Kenyon, R., Vershik, A.: Arithmetic construction of sofic partitions of hyperbolic toral automorphisms. Ergodic Theory Dyn. Syst. 18(2), 357-372 (1998)

64. Lalley, S.P., Gatzouras, D.: Hausdorff and box dimensions of certain self-affine fractals. Indiana Univ. Math. J. 41(2), 533-568 (1992)

65. Le Borgne, S.: Un codage sofique des automorphismes hyperboliques du tore. In: Séminaires de Probabilités de Rennes. Publ. Inst. Rech. Math. Rennes, vol. 1995, p. 35. University of Rennes 1, Rennes (1995)

66. Le Borgne, S.: Un codage sofique des automorphismes hyperboliques du tore. C.R. Acad. Sci. Paris Sér. I Math. 323(10), 1123-1128 (1996)

67. Le Borgne, S.: Un codage sofique des automorphismes hyperboliques du tore. Bol. Soc. Brasil. Mat. (N.S) 30(1), 61-93 (1999)

68. Lind, D., Marcus, B.: An Introduction to Symbolic Dynamics and Coding. Cambridge University Press, Cambridge (1995)

69. Livshits, A.N.: On the spectra of adic transformations of markov compacta. Russ. Math. Surv. 42, 222-223 (1987)

70. Livshits, A.N.: Some examples of adic transformations and automorphisms of substitutions. Sel. Math. Sov. 11(1), 83-104 (1998). Selected translations

71. Lothaire, M.: Applied Combinatorics on Words. Encyclopedia of Mathematics and its Applications, vol. 105. Cambridge University Press, Cambridge (2005)

72. Luck, J.M., Godrèche, C., Janner, A., Janssen, T.: The nature of the atomic surfaces of quasiperiodic self-similar structures. J. Phys. A 26(8), 1951-1999 (1993)

73. Mauldin, R.D., Williams, S.C.: Hausdorff dimension in graph directed constructions. Trans. Am. Math. Soc. 309(2), 811-829 (1988)

74. McMullen, C.: The Hausdorff dimension of general Sierpiński carpets. Nagoya Math. J. 96, 1-9 (1984)

75. Minervino, M., Steiner, W.: Tilings for Pisot beta numeration. Indag. Math. (N.S.) 25(4), 745-773 (2014)

76. Moody, R.V.: Model sets: a survey. In: Axel, F., Dénoyer, F., Gazeau, J.-P. (eds.) From Quasicrystals to More Complex Systems. Centre de Physique des Houches, vol. 13, pp. 145-166. Springer, Heidelberg (2000)

77. Praggastis, B.: Numeration systems and Markov partitions from self-similar tilings. Trans. Am. Math. Soc. 351(8), 3315-3349 (1999)

78. Queffélec, M.: Substitution Dynamical Systems-Spectral Analysis, vol. 1294. Springer, Heidelberg (1987)

79. Rauzy, G.: Nombres algébriques et substitutions. Bull. Soc. Math. Fr. 110(2), $147-178(1982)$

80. Robinson, Jr., E.A.: Symbolic dynamics and tilings of $\mathbb{R}^{d}$. In: Symbolic dynamics and its applications. Proc. Sympos. Appl. Math., Amer. Math. Soc., vol. 60, pp. 81-119, Providence, RI (2004)

81. Roy, D.: Approximation to real numbers by cubic algebraic integers. II. Ann. Math. (2) 158(3), 1081-1087 (2003)

82. Rudin, W.: Some theorems on Fourier coefficients. Proc. Am. Math. Soc. 10, 855859 (1959)

83. Senechal, M.: What is... a quasicrystal? Not. Am. Math. Soc. 53(8), 886-887 (2006)

84. Siegel, A., Thuswaldner, J.M.: Topological properties of Rauzy fractals. Mém. Soc. Math. Fr. (N.S.) 118, 140 (2009) 
85. Sirvent, V.F., Solomyak, B.: Pure discrete spectrum for one-dimensional substitution systems of Pisot type. Canad. Math. Bull. 45(4), 697-710 (2002). (Dedicated to Robert V. Moody)

86. Sirvent, V.F., Wang, Y.: Self-affine tiling via substitution dynamical systems and Rauzy fractals. Pac. J. Math. 206(2), 465-485 (2002)

87. Thue, A.: Über unendliche Zeichenreihen. Norske Vid. Selsk. Skr. Mat. Nat. Kl. 37(7), 1-22 (1906)

88. Thue, A.: Über die gegenseitige Lage gleicher Teile gewisser Zeichenreihen. Norske Vid. Selsk. Skr. Mat. Nat. Kl. 43(1), 1-67 (1912)

89. Thurston, W.P.: Groups, tilings and finite state automata. Lectures notes distributed in conjunction with the Colloquium Series, in AMS Colloquium lectures (1989)

90. Thuswaldner, J.M.: Unimodular Pisot substitutions and their associated tiles. Journal de Theorie des Nombres de Bordeaux 18(2), 487-536 (2006)

91. Veech, W.A.: Interval exchange transformations. J. Anal. Math. 33, 222-272 (1978)

92. Yasutomi, S.-I.: On Sturmian sequences which are invariant under some substitutions. In: Number theory and its Applications (Kyoto, 1997). Dev. Math., vol. 2, pp. 347-373. Kluwer Academic Publishers, Dordrecht (1999) 